\title{
IMPLICAÇÕES DA PANDEMIA DE COVID-19 EM CRIANÇAS E ADOLESCENTES COM TRANSTORNO DO ESPECTRO AUTISTA
}

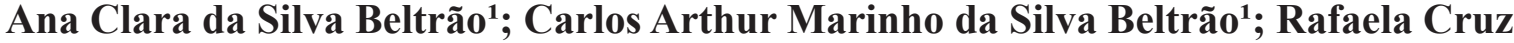 de Oliveira'; ${ }^{1}$ Mayra Cristina Cavalcante Campos'; Sofia Rodrigues Gonçalves'; Beatriz}

Albuquerque Bom im'.

${ }^{1}$ Acadêmico de Medicina, Universidade do Estado do Amazonas (UEA), Manaus, Amazonas. DOI: 10.47094/IICNNESP.2021/171

\section{RESUMO}

Introdução: Em virtude das características inerentes ao Transtorno do Espectro Autista (TEA), as mudanças impostas pela pandemia de COVID-19 poderiam condicionar sofrimento emocional e a exacerbação de problemas comportamentais nesses indivíduos. Objetivo: apresentar uma revisão narrativa da literatura acerca das implicações da pandemia de COVID-19 em crianças e adolescentes com TEA. Metodologia: Foram realizadas pesquisas digitais em livros-texto e na base de dados PubMed, utilizando-se de descritores como "autism", "pandemia" e "COVID-19". Fundamentação teórica: As interrupções nos serviços impactaram negativamente nos sintomas, comportamentos ou outros desafios do TEA, além disso, crianças com problemas comportamentais prévios à pandemia tiveram uma tendência 2,16 vezes maior de exibir problemas comportamentais mais intensos e 1,67 vezes mais frequentes do que aquelas sem problemas comportamentais prévios. Considerações inais: Com o surto da pandemia de COVID-19, crianças e adolescentes sofreram implicações que perpassaram desde aspectos educacionais e terapêuticos a aspectos comportamentais e emocionais.

PALAVRAS-CHAVE: Sofrimento emocional. Problemas comportamentais. Isolamento social.

ÁREA TEMÁTICA: Saúde Física e Mental.

\section{INTRODUÇÃO}

Transtorno do Espectro Autista (TEA) é um diagnóstico definido no Manual Diagnóstico e Estatísticos de Transtornos Mentais como déficits na comunicação e interação sociais, e padrões de interesses e comportamentos repetitivos (DSM-5, 2014). Tais características acabam por condicionar os indivíduos com TEA à uma preferência por ambientes previsíveis, levando-os à tendência de experienciar sofrimento psicológico se submetidos a mudanças bruscas e significativas em suas rotinas (COLIZZI et al., 2020), podendo até desencadear medo, pânico e comportamentos de birra (SADOCK; SADOCK; RUIZ, 2017).

Mediante a emergência da pandemia de COVID-19 e a obrigatoriedade do isolamento social imposto pelas autoridades, houve a interrupção de diversos serviços presenciais e o fechamento de escolas, incluindo aqueles direcionados a esse grupo. 
O fechamento temporário desses serviços poderia lhes condicionar um risco aumentado de resultados negativos, haja vista que escolas, além de prestarem serviços educacionais, também são responsáveis pela entrega de diversas modalidades de terapias e intervenções comportamentais, bem como o suporte psicológico (WHITE et al., 2021). Além disso, todas essas mudanças, incluindo a adoção de medidas de higiene, o isolamento social, poderiam condicionar a exacerbação de problemas comportamentais e o surgimento de sofrimento emocional em indivíduos com TEA (SBP, 2020).

Portanto, considerando a atual conjuntura e a vulnerabilidade dessa população mediante a situações desafiadoras, o presente estudo visa apresentar uma revisão narrativa da literatura acerca das implicações da pandemia de COVID-19 em adolescentes e crianças com TEA.

\section{METODOLOGIA}

O presente artigo trata-se de uma revisão narrativa de literatura. Para a sua elaboração foram realizadas buscas digitais em livros-texto e na base de dados PubMed, por meio dos descritores "autism", "COVID-19" e "pandemia". Durante a pesquisa no PubMed, foram aplicados alguns filtros específicos que facilitaram a seleção de artigos à cerca da temática, tais como "texto completo grátis" e data de publicação de "1 ano". Foram incluídos artigos que tenham sido publicados em português, inglês e espanhol, que tenham sido disponibilizados gratuitamente na íntegra e com data de publicação de 1 ano, bem como artigos cujos sujeitos do estudo fossem crianças e adolescentes. Sendo, portanto, excluídos artigos cujos sujeitos fossem exclusivamente adultos, artigos que não apresentavam metodologia e que não contemplaram plenamente a temática do estudo. Ao todo foram incluídos 1 nota técnica da SBP (Sociedade Brasileira de Pediatria), 2 livros-textos e 4 artigos no estudo.

Após a análise do acervo desenvolvido, a construção do texto seguiu-se mediante avaliação crítica do conteúdo disponibilizado nas publicações.

\section{FUNDAMENTAÇÃO TEÓRICA}

Os indivíduos com TEA são vulneráveis ao surgimento de sintomas psiquiátricos no decorrer da pandemia de COVID-19, quer seja pela forte inflexibilidade e, portanto, dificuldade em gerir novas situações, quanto pela maior suscetibilidade decorrente de transtornos psiquiátricos comórbidos, frequentemente prevalentes nessa população. Entretanto, com a significativa redução das demandas acadêmicas e sociais exigidas, esses indivíduos potencialmente poderiam se beneficiar do isolamento social e evidenciar melhora em seu estado de saúde físico e mental (LUGO-MARÍN et al. 2020).

O tratamento empregado no TEA consiste em uma abordagem multidisciplinar de metodologias intensivas ou menos intensivas (SBP, 2020). Em casos de crianças mais novas, onde as terapias empregadas consistem em jogos interativos e na ABA (Applied Behaviour Analysis), muitas horas são dispendidas, como cerca de 20 a 40 horas semanais. Potencialmente, o cessamento das terapias e dos serviços prestados à essa população, bem como as mudanças na rotina, poderiam 
agravar os sintomas do transtorno, aumentar os desafios comportamentais exigidos e resultar em um impacto negativo na saúde mental dessas crianças (WHITE et al. 2021).

White et al. (2021), em estudo realizado com 3502 pais e cuidadores de crianças com TEA registradas na coorte SPARK, com o intuito de investigar o impacto de COVID-19 nos serviços prestados e a perspectiva dos pais/cuidadores sobre a eficácia da adaptação desses serviços, bem como o impacto dessas interrupções nos indivíduos e suas famílias, puderam constatar que independentemente da idade, a maioria desses indivíduos tiveram suas terapias interrompidas, sendo essas: educação especial (80\%), terapia fonoaudiológica (88\%), terapias físicas e ocupacionais (84\%) e ABA (77\%). Ao investigar o recebimento de serviços online/adaptados, a maioria dos pais/ cuidadores relataram o não recebimento desses serviços. Além disso, 64\% dos entrevistados relataram que as interrupções nos serviços impactaram moderadamente ou severamente de forma negativa nos sintomas, comportamentos ou outros desafios do TEA de seus filhos.

Colizzi et al. (2020) em uma pesquisa online com 527 pais e tutores, buscaram investigar o impacto da pandemia de COVID-19 em crianças com TEA no Norte da Itália, se e quais características clínicas e sociodemográficas poderiam prever implicações negativas, e caracterizar de forma narrativa as necessidades das mesmas e suas famílias. Os autores identificaram dificuldade dos pais em gerir a refeição dos filhos (23\%), autonomias (31\%), tempo livre $(78,1 \%)$, bem como atividades estruturadas $(75,7 \%)$, sendo estas consideradas mais difíceis de gerenciar comparativamente ao período antecedente à pandemia. Uma proporção considerável de pais relatou que os problemas comportamentais dos filhos foram mais intensos $(35,5 \%)$ e mais frequentes $(41,5 \%)$ que antes do surto da pandemia. Ao avaliar a existência de preditores de impacto negativo, os autores detectaram que em comparação com crianças sem problemas comportamentais prévios, aquelas que os detinham previamente tiveram uma tendência 2,16 vezes maior de exibir problemas comportamentais mais intensos, e 1,67 vezes maior de exibir problemas comportamentais mais frequentes. Houve também associação entre não receber apoio escolar indireto e a probabilidade do aumento de problemas comportamentais mais intensos. Entretanto, idade mais avançada e morar com pais solteiros surpreendentemente se mostrou um fator protetivo, sendo associado com uma probabilidade menor de exibir problemas comportamentais mais intensos.

Nonweiler et al. (2020) ao investigar a prevalência de problemas emocionais e comportamentais, bem como fatores associados aos mesmos, em 453 crianças e adolescentes com transtornos do neurodesenvolvimento nos primeiros estágios da pandemia de COVID-19, identificaram que aquelas com transtorno do neurodesenvolvimento tiveram uma prevalência superior de sintomas emocionais quando comparadas com crianças neurotípicas (42\% vs. 15\%), problemas comportamentais mais elevados (28\% vs. $9 \%$ ), além de comportamentos pró-sociais reduzidos ( $54 \%$ vs. $22 \%$ ). Ademais, meninas com TEA evidenciaram mais sintomas emocionais em comparação com meninos $[$ média(DP) $=7,2(2,5)$ vs. 5,2(2,8)].

Lugo-Marín et al. (2021) em um estudo que avaliou o impacto psicológico do confinamento em crianças/adolescentes e adultos com TEA, constataram que crianças e adolescentes demonstraram melhora em seu status psicopatológico, porém sem significância estatística. A melhora também pode 
ser observada no status psicopatológico das crianças e adolescentes de acordo com seu nível de gravidade (nível 1 e 2), onde aquelas do nível 2 exibiram uma melhora superior àquelas do nível 1. Sendo destacada a possibilidade da melhora em virtude aos ajustes farmacológicos. Além disso, as crianças do nível 1 demonstraram benefício com o isolamento social nos sintomas de abstinência/ depressão.

\section{CONSIDERAÇÕES FINAIS}

Após o surto da pandemia de COVID-19, crianças e adolescentes sofreram implicações significativas em suas vidas, perpassando desde aspectos educacionais e terapêuticos a aspectos comportamentais e emocionais. Observou-se que a interrupção das escolas e serviços terapêuticos impactaram negativamente no comportamento ou outros desafios de indivíduos com TEA. Aquelas crianças cujos problemas comportamentais estavam presentes previamente ao início da pandemia, tiveram uma probabilidade maior de evidenciar problemas comportamentais mais intensos e frequentes. Entretanto, também foi constatado que algumas crianças se beneficiaram da obrigatoriedade do distanciamento social.

\section{PRINCIPAIS REFERÊNCIAS}

COLIZZI, Marco et al. Psychosocial and Behavioral Impact of COVID-19 in Autism Spectrum Disorder: an online parent survey. Brain Sciences, [S.L.], v. 10, n. 6, p. 341-355, 3 jun. 2020. MDPI AG. http://dx.doi.org/10.3390/brainsci10060341. Disponível em: https://dx.doi. org/10.3390\%2Fbrainsci10060341. Acesso em: 20 abr. 2021.

Manual Diagnóstico e Estatístico de Transtornos Mentais: DSM-5. 5. ed. Porto Alegre: Artmed, 2014.

NONWEILER, Jacqueline et al. Prevalence and Associated Factors of Emotional and Behavioural Difficulties during COVID-19 Pandemic in Children with Neurodevelopmental Disorders. Children, [S.L.], v. 7, n. 9, p. 128-132, 4 set. 2020. MDPI AG. http://dx.doi.org/10.3390/children7090128. Disponível em: https://dx.doi.org/10.3390\%2Fchildren7090128. Acesso em: 22 abr. 2021.

WHITE, L. Casey et al. Brief Report: impact of covid-19 on individuals with asd and their caregivers. Journal Of Autism And Developmental Disorders, [S.L.], p. 1-8, 2 jan. 2021. Springer Science and Business Media LLC. http://dx.doi.org/10.1007/s10803-020-04816-6. Disponível em: Journal of Autism and Developmental Disorders. Acesso em: 21 abr. 2021. 